\title{
INCREASE IN VORTEX CAVITATOR EFFICIENCY BY WORKING AREA COOLING METHOD
}

\author{
Evgeniy Ivanov \\ Nizhny Novgorod State Agricultural Academy, Russia \\ ivanov.e.g@mail.ru
}

\begin{abstract}
Acoustic cavitation treatment of liquid media accelerates well-known technological processes and it makes feasible the processes which seemed to be impossible primarily. Besides, it changes the properties of water by giving it a biological activity. Therefore, these technologies have large perspectives to use them in agriculture, such as in seed germination, crop watering, product processing, environment restoring. This technology represents the passing of 2.5-3 bar intensity sound waves through the liquid. As a result, the liquid breaks in the vacuum phase. But in the manometrical phase, there is a collapse of these breaks. However, such treatment in vortex cavitators (liquid whistles) is accompanied by an increase in temperature. It provokes an intensive flow of steam and dissolved air from the water to the cavity, which is formed in the vacuum phase. Consequently, the final volume of such caverns in the manometric phase collapse will be larger, and the energy density in each space point will be lower than the threshold value. As a result, a required amount of transformation of forms of energy (including the forms that provoke the change of water nano-structures) will not take place. Therefore, at high-temperature conditions, the efficiency of the processes is low, and at lowtemperature conditions the treatment is incomplete, because it is achieved by reducing the time of water treatment in the cavitator. Cooling of the active cavitation zone of the cavitator has increased the perspectives and efficiency of its use - water takes on new properties in larger volumes, and technical operations run with a greater productivity and with an improved quality.
\end{abstract}

Keywords: liquid, cavitation cavity, evaporation, collapse, cooling.

\section{Introduction}

The specificity of acoustic cavitation technologies is the nano-size of their functioning. For example, in decomposition and mixing operations it may be achieved, if it is possible to destroy the unity of liquid and pollutant in local space points. The shearing deformation is also necessary. If other process tasks are realized, the change of energy forms (magnetic [1;2], thermal, mechanical energy of deformations, electromagnetic, etc.) is very important. But these possibilities may be realized, only if the demanded energy density level in the cavity collapse is achieved. It is caused by the final volume of the caverns while they are collapsed.

So, if a full vacuum of the cavity in the final stage of the collapse the counter surfaces would link up and the energy density would tend to infinity. But actually, when the cavity appears and grows, the liquid evaporates the gases, which were dissolved in this liquid before transfer [3; 4]. When the cavity walls during the collapse approach the highest volume, it has its final value. At that, the lowest value causes a higher level of the energy density and it means a higher temperature and tension level. If the final volume in the collapsed cavity is higher (it takes place in high temperature mode), the temperature and tension do not achieve the demanded level. The process flow parameters become lower.

The method of the increase in general pressure in the working area is used in order to prevent the above-mentioned processes. It reduces the processes of the liquid evaporation and degassing and it increases by tens of percent the effectiveness of these technologies [3;5]. However, this method demands a higher hermeticity of the cavitators. It provokes the decrease in its reliability. It cannot compensate the costs caused by a higher temperature in the high temperature modes.

Another method is suggested in this paper. It is cooling of the working area. It will reduce the vaporization property and the degassing of working liquid. Consequently, in this case, a higher result may be achieved with lower costs. These methods may be used independently, as well as in combination with each other.

\section{Materials and methods}

The degassing and vaporization processes are the main reasons, which provoke the decrease in effectiveness of the acoustic cavitation processes in high temperature modes. They start in the phase of the cavity appearance and growth. 
Vaporization - the process of abstraction of the more rapid molecules from the surface of the liquid and of their transformation in the vapor [6]. The vaporization process becomes more intensive, when the temperature increases, because in this case the number of rapid molecules increases. The decrease in the tension provokes: the decrease in the liquid density, the increase in the distance between the molecules, the weakening of the connection between the molecules, the increase in the weekly confined molecule number. All of these things contribute to the activation of the vaporization.

It is possible to use the following famous formula to assess the vacuum water vaporization rate.

$$
G_{\max }=\frac{f\left(p_{0}-p\right)}{g \sqrt{2 \pi R T / M}}=e\left(p_{0}-p\right) \sqrt{\frac{M}{2 \pi R T}},
$$

where $p_{0}$ - vapor tension of the vaporized substance;

$p$ - partial pressure of water vapor;

$R$ - universal gas constant;

$M$ - molecular weight of the substance;

$e$-empirical constant, it takes into account blocking of the free surface.

Relative to water as a concrete liquid this formula takes the following form

$$
W=e A\left(p_{0}-p\right) \mathrm{g} \cdot \mathrm{s}^{-1},
$$

where $A$ - surface of vaporization.

Relative to water vaporizing in the vacuum atmosphere

$$
W=e A p_{0}
$$

The influence of the tension is assessed by the absence of the $p$ component, the assessment of the temperature influence is made based on the analyses of its influence on the equilibrium vapor pressure of water as the vaporized liquid, i.e.

$$
P=0.128 t^{3}-4.58 t^{2}+171.07 t-140.68 \mathrm{~Pa} .
$$

consequently,

$$
W=e A\left(0.128 t^{3}-4.58 t^{2}+171.07 t-140.68\right) \mathrm{g} \cdot \mathrm{s}^{-1} .
$$

The speed of the change of the level in water vaporization

$$
h=3.527 \cdot 10^{-8}\left(0.128 t^{3}-4.58 t^{2}+171.07 t-140.68\right) \mathrm{mm} \cdot \mathrm{s}^{-1} .
$$

Based on this equation, it is possible to define the speed of vaporization $\left(\mathrm{mm} \cdot \mathrm{s}^{-1}\right)$ from the surface of water to vacuum in different temperatures (Fig.1).

If the cavity size $d=0.4 \mathrm{~mm}$, the time of its existence $t=0.0004 \mathrm{~s}$ (Fig.2) for the frequency $f=2 \mathrm{kHz}$, the volume of the water vaporized from the surface of the environment board will be $w=\pi d^{2} \cdot t \cdot h$. The dependency on different temperatures may be described by a similar formula Fig. 1 .

$$
w=1.7635 \cdot 10^{-11}\left(0.128 t^{3}-4.58 t^{2}+171.07 t-140.68\right) \mathrm{mm}^{3} .
$$

While the cavity is collapsed the vapor phase will occupy the volume of the vaporized liquid. Its volume may be defined based on equation (7).

Degassing. Water, like other liquids, contacting with a gas completely dissolves it [7]. It is necessary to take into account that water is delivered to the cavitation units of the industrial enterprises by water supply lines interplayed over the earth surface, its temperature $t \approx 8-100^{\circ}$, its pressure $p \approx$ 6 bar. It contains air locks, which provokes its saturation by air (Fig. 3).

$$
C_{0}=112 \frac{24}{17}=158 \mathrm{~m}^{3} \cdot 1000 \mathrm{~m}^{-3}
$$

If such water passes through the cavitation area in $t \approx 90^{\circ}$, all the contained in the water air will escape and it will be a self-consistent gas phase. If the temperature value in the cavitation area is 
lower, the proportion of the air escaped to a free state from maximum saturated water $\left(t \approx 8 \div 100^{\circ}, p\right.$ $\approx 6$ bar), will be lower (Fig. 4).

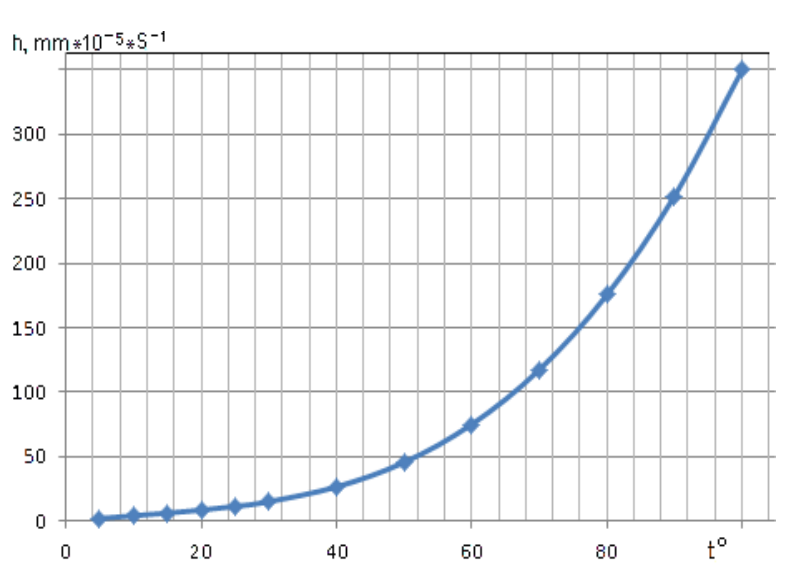

Fig. 1. Dependence of vacuum water vaporization rate on the temperature; $\mathrm{mm} \cdot 10^{-5} \cdot \mathrm{s}^{-1}$

a)

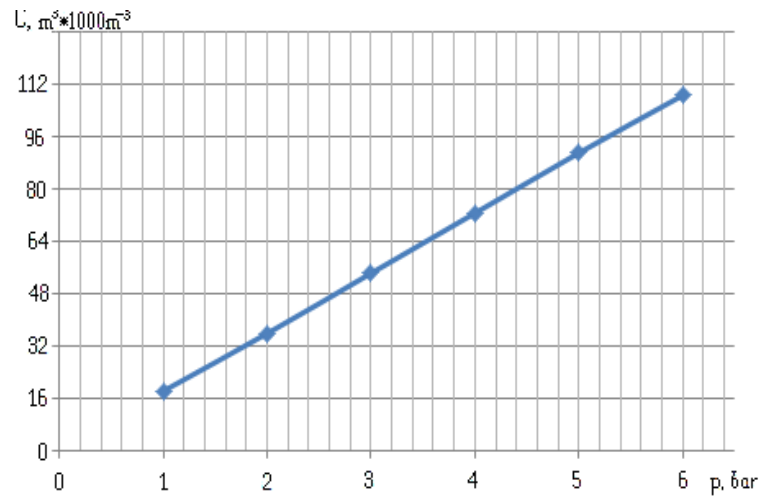

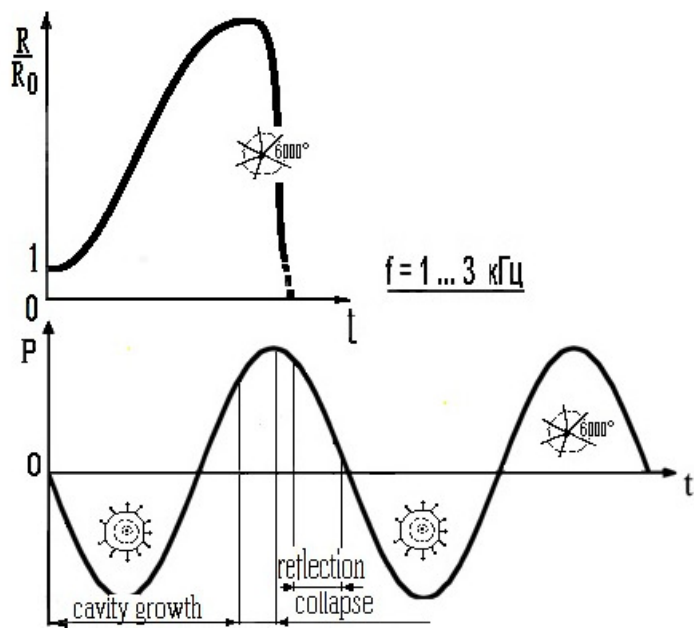

Fig. 2. Change of the cavity size over time

b)

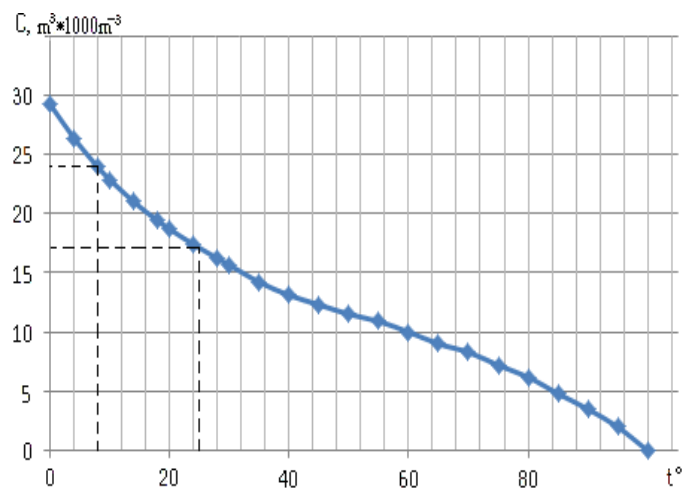

Fig. 3. Concentration of the air dissolved in water $\mathrm{m}^{3} * 1000 \mathrm{~m}^{-3}$ depending on: $\mathrm{a}$ - pressure, bar, $\left(\mathrm{t}=25^{\circ} \mathrm{C}\right) ; \mathrm{b}$ - temperature, ${ }^{\circ} \mathrm{C},(\mathrm{p}=1$ bar $)$

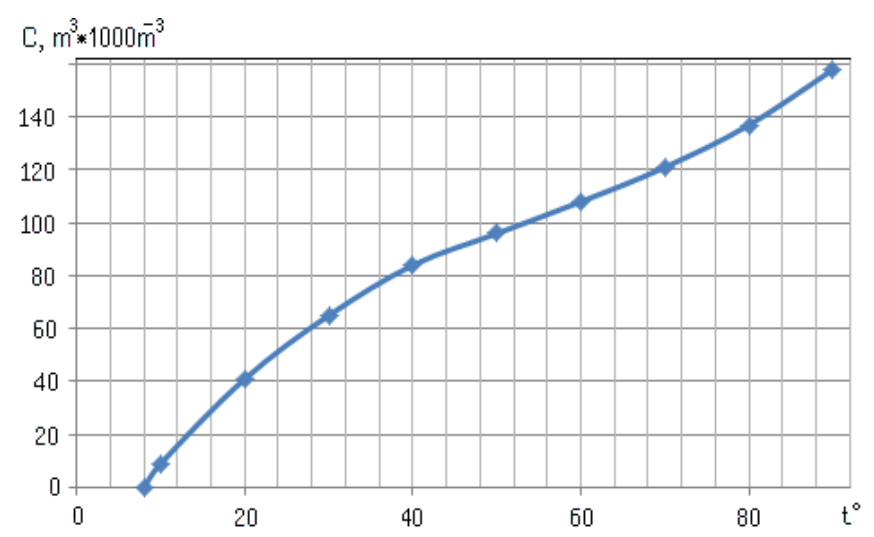

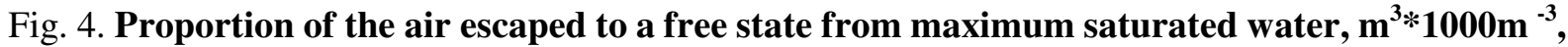
depending on the temperature ${ }^{\circ} \mathrm{C},\left(t \approx 8 \div 100^{\circ}, p \approx 6 \mathrm{bar}\right)$

The processes of vaporization and degassing in the cavity begin at the same time with the appearance of the cavity itself, i.e. of the free surface of the phase interface. The degassing process acts from the cavity surface and its near-surface layer. If the cavity enlarges, each its new surface absorbs by its volume. Consequently, the volume in the liquid massive, which is equal to the volume of the cavity in its maximal enlargement 


$$
W=\frac{4}{3} \pi \frac{d^{3}}{8}=\pi \frac{1}{6} 0.4^{3}=0.0335 \mathrm{~mm}^{3},
$$

may be considered as a source of degassing. So, the volume of the air escaped from the solubility, depending on the temperature of the cavitation area, may be defined as a product of the cavity volume by the proportion of the air in it Fig. 4.

All the processes mentioned above take place in the cavity appearance and growth phases. As for the collapse phase, there is a good reason to consider the external input of the cavity walls moving because of manometrical pressure on the internal spherical concentric barrier. The Rayleigh method [3] (considering that the kinetic energy of wall movement is equal to the work done by pressure on the cavity).

$$
p_{2}=0.163 p_{1} \frac{R_{1}^{3}}{R_{2}^{3}}
$$

This formula is driven to the form

$$
p_{2} W_{2}=0.163 p_{1} W_{1}=C_{1},
$$

where $R_{1}, W_{1}$ - radius and volume of the cavity in the final stage of the growth;

$R_{2}, W_{2}$ - radius and volume of the cavity in the final stage of the collapse;

$p_{1}$ - external pressure in the cavitation area;

$p_{2}$ - pressure in the cavity in the final stage of collapse.

If the size of the cavity decreases, the forces of hydrodynamic pressure will be compensated for the forces of pressure of the air escaped from the solubility (taking into account the water vapor transformed to the water again in inconsiderable pressure range at the beginning of the pressure process). So, if the isometric regularity is admissible, then

$$
\left(W_{1}-W_{\text {wat }}\right) p_{1}^{*}=\left(W_{2}-W_{\text {wat }}\right) p_{2}=C_{2},
$$

where $W_{w o t}-$ volume of vapored water in the cavity appearance and growth phase;

$p_{1}{ }^{*}$ - pressure of the degassed air in the cavity before its collapse, $p_{1}{ }^{*}=C p_{a t} ;(\mathrm{C}$ from Fig. 4).

By solving simultaneous equations between (10) and (11), we receive

$$
p_{2}=\frac{C_{1}-C_{2}}{W_{\text {wat }}} ; W_{2}=\frac{C_{1}}{p_{2}}
$$

The $p_{2}$ calculation data for different values of the temperature are represented as a characteristic curve (Fig. 5).

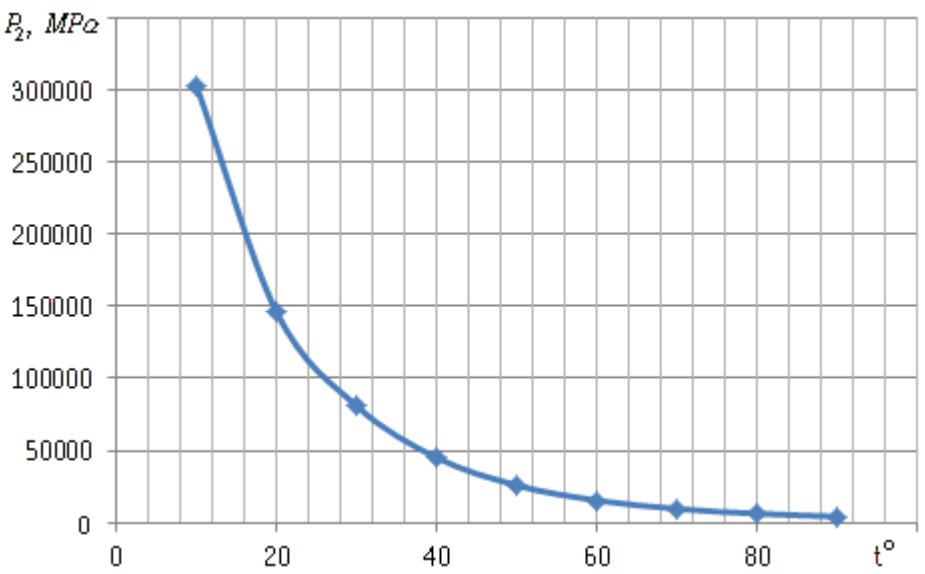

Fig. 5. Interpretation of calculation data of the pressure (energy density) in the cavity in the final stage of the collapse in different temperatures of the cavitator work 
The received dependency (Fig. 5) shows that the pressure creating while the cavity is collapsed is considerably higher in low temperature modes than that in the high temperature modes. It proves the domination of the factors of vaporizing and water degassing in the functioning of these cavities.

An investigational study was made in order to verify the studied hypothesis. It was necessary to determine how the water heating value $\Delta t$ depends on the transit exemption value $q(\Delta t=f(q))$ in the following cases:

- cooling is absent;

- cooling is made by streaming water contained in aqueduct.

The exemption $q$ is measured with a measuring container and stopwatch, the temperature is measured with a digital infrared pyrometer CM-320 manufactured in 2018.

The heat power $N_{\text {term }}=f(q$ is defined by the $\Delta t=f(q)$ dependency representing the transit exemption $q$ and temperature differential $\Delta \mathrm{t}$ product taking into account the dimensions.

The dependency of the heat power $\mathrm{N}_{\text {term }}$ on the heating temperature $\Delta t$ was defined using the formulas $\Delta t=f(q)$ and $N_{\text {term }}=f(q)$ in each transit exemption value $q$.

Data on thermal index cavitator is summarized on Table 1.

Table 1

Thermal index cavitator

\begin{tabular}{|c|c|c|c|c|c|c|c|c|}
\hline $\begin{array}{c}\tau \text { prot., } s \\
(W= \\
\left.1 \cdot 10^{-2} \mathrm{~m}^{3}\right)\end{array}$ & $\begin{array}{l}Q^{* 10^{-6}}, \\
\mathbf{m}^{3} * \mathbf{s}^{-1}\end{array}$ & $\begin{array}{c}\Delta t^{\mathbf{o}} \\
\text { prot. }\end{array}$ & $\begin{array}{c}N \\
\text { prot., } \\
\text { kW }\end{array}$ & $\begin{array}{c}\Delta t^{\mathrm{o}} \\
\text { model }\end{array}$ & $\begin{array}{c}N \\
\text { model, } \\
\text { kW }\end{array}$ & $\begin{array}{c}t_{\text {cool }}-\mathbf{8}^{\mathbf{0}} \\
\operatorname{model}\left(\boldsymbol{q}_{\text {cool }}=\right. \\
\left.2 \cdot 10^{-4} \mathrm{~m}^{3} \cdot \mathrm{s}^{-1}\right)\end{array}$ & $\begin{array}{c}N_{\text {cool }} \\
\text { model, } \\
\text { kW }\end{array}$ & $\begin{array}{c}N_{f u l l} \\
\text { model, } \\
\mathbf{k W}\end{array}$ \\
\hline 1428 & 7 & 110 & 3.2 & 60 & 1.8 & 10.0 & 9.5 & 10.3 \\
\hline 435 & 23 & 60 & 5.8 & 45 & 4.3 & 6.9 & 5.8 & 10.1 \\
\hline 217 & 46 & 38 & 7.3 & 30 & 5.8 & 5.0 & 4.2 & 10.0 \\
\hline 147 & 68 & 28 & 8.0 & 25 & 7.3 & 3.1 & 2.6 & 9.9 \\
\hline 111 & 90 & 24 & 8.9 & 21.7 & 8.2 & 2.2 & 1.8 & 10.0 \\
\hline 86 & 115 & 20 & 9.5 & 9.3 & 9.3 & 0.8 & 0.7 & 10.0 \\
\hline
\end{tabular}

\section{Results and discussion}

The above mentioned dependencies are proved by the experiments testing the heat generation of the vortex cavitator. So, the temperature characteristic of the cavitator shows that the temperature of the transfer stream through the cavitator depends on the transfer stream q and its dependency represents a hyperbolic law (Fig. 6a).

The dependency of the heat power generated by the cavitator on the temperature mode (Fig. 6b) based on the temperature curve (Fig. 6a) shows a grand similarity with that of the tension in the collapse area relative to different temperature modes (Fig. 5). So, the tension value characterizing in this case the energy density determines the effectiveness of the periodic cavitation process and it depends on the process conditions.

Consequently, it seems to be expedient to cool the cavitation area of the unit, for example, by the cylinder jacket with cooling liquid flow or gas.

The author made an experimental test on the test bench (Fig. 7) containing a cylinder jacket for cooling the vortex chamber and resonator.

The research results proved that the decision was right: ineffective and low consumed mode of the working process became effective because of cooling and even the effectiveness of the high consumed modes increases, because the number of the working liquid cavitation treatment cycle increases (Fig. 8).

So, the heat power of the cavitator reference design depends on the transit exemption value and its dependency represents the parabolic general equation of the second degree

$$
A x^{2}+2 B x y+C y^{2}+2 D x+2 E y+F=0,
$$

i.e. its effectiveness approaches zero in the area of lower water flow (curve 1, curve 3 ), because this mode is accompanied with higher heating. If the cavitation area is cooled with aqueduct streaming 
water in the cylinder jacket (Fig. 7), the generated heat value increases abruptly. But in this case, this heat is taken through two ways:

- with transit exemption;

- with cooled water.

The total (curve 2) value of the heat power is considerably higher than that of the prototype (curve 1).

a)

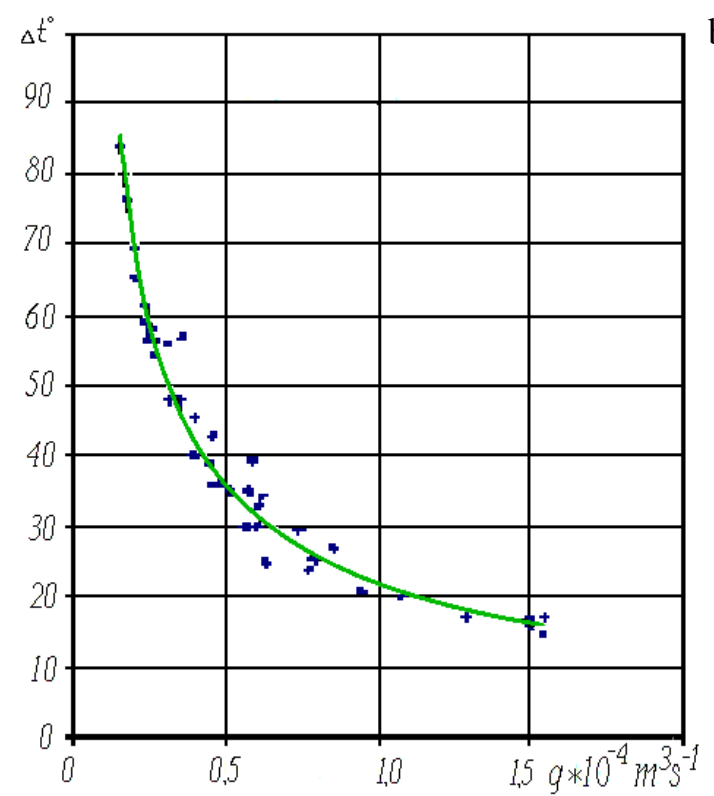

b)

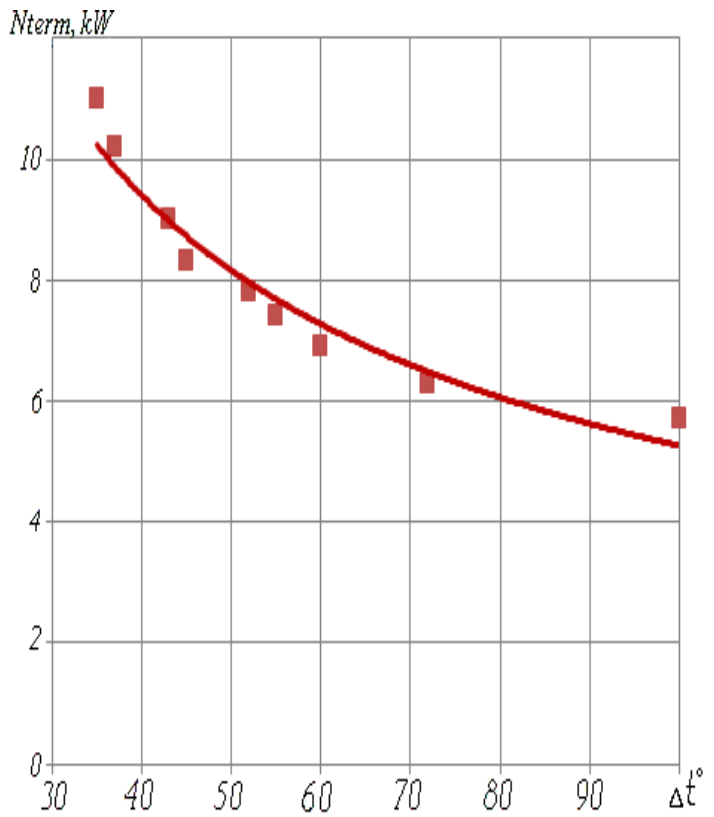

Fig. 6. Heat characteristic of the vortex cavitator: a - temperature curve $\Delta t=f(q)$; b - thermal characteristic $N_{\text {term }}=f(\Delta t)$

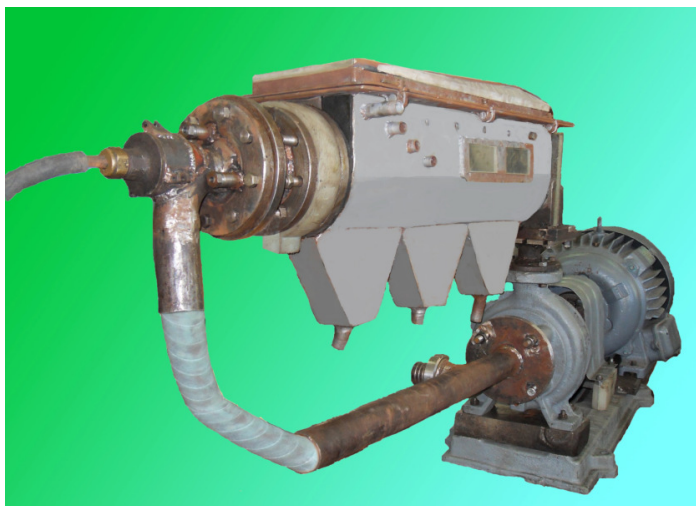

Fig. 7. Test bench of the vortex cavitator with cylinder jacket

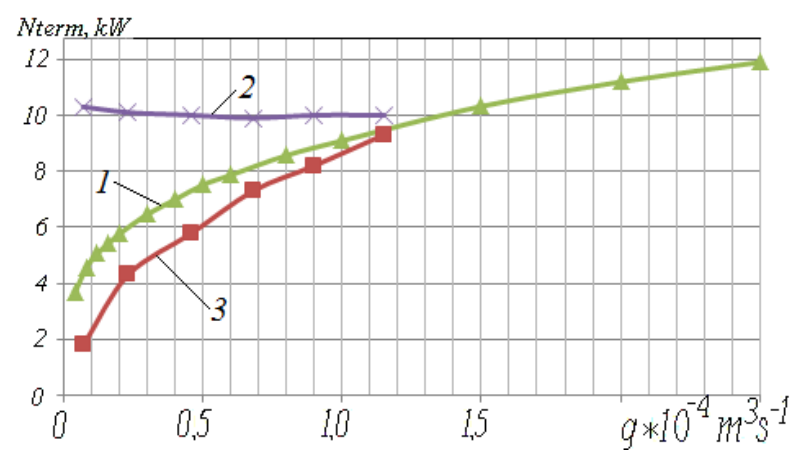

Fig. 8. Influence of cooling on the heat characteristic $N_{\text {term }}=f(q)$ of the vortex cavitator

This engineering proposal offers an additional important source- the increase in effectiveness of periodic cavitation of modes, in which there are higher numbers of cycles, which the working liquid passes through the working zones. On its turn, it will provide a more detailed treatment of water, as well as of other components treated together with it. Thereby, it is possible to suppose that all the functions discovered in the cavitator may be realized with a higher quality and productivity, the additional discovered properties of water $[8 ; 9 ; 10]$ will get additional manifestation and reinforcement, because it becomes possible to get a higher number of full collapses in the cavitation treatment cycle.

It is possible to use the described method in vortex chambers working on gases $[11 ; 12]$ in order to manage their working process. 


\section{Conclusions}

1. It is shown that vaporization and water degassing to the internal parts of the cavities make a negative influence on cavitation processes.

2. It is established that these negative phenomena become stronger, when the operating temperature increases while the cavitator is functioning.

3. The analogy between the dependency of the heat power generated by the cavitator with that of the tension in the cavity collapse on the temperature mode is discovered by the cavitator.

4. The technical solution representing the cooling of cavitator working area by a water jacket is suggested.

5. The realization of the suggested measures will permit to increase the deepness, completeness, and the productivity of liquid-saturated medium cavitation.

6. The use of a modernized cavitator will permit to discover most completely and on a higher level the earlier unknown properties of water.

\section{References}

[1] Margulis M.A. Sound chemical reactions and sonoluminescence. Monograph. Moscow: Chemistry, 1986. 288 p.

[2] Ivanov Y.G., Saharov A.N. On the possibility of using physical fields from acoustic cavitation in liquid environments in the processing of seeds by dry technology. Proceedings of V International scientific congress "Agricultural machinery", June 1, 2018, Burgas, Bulgaria, pp. 163-167.

[3] Agranat B.A., Dubrovin M.N., Havskiy N.N., etc. Fundamentals of physics and technology of ultrasound. Moscow: Graduate School, 1987. 352 p.

[4] Qiu S., Ma X., Huang B. etc. Numerical simulation of single bubble dynamics under acoustic standing waves. Ultrasonics Sonochemistry, vol. 49, 2018, pp. 196-205.

[5] Wu P., Bai L., Lin W. etc. Mechanism and dynamics of hydrodynamic-acoustic cavitation (HAC). Ultrasonics Sonochemistry, vol. 49, 2018, pp. 89-96.

[6] Chemistry Handbook 21. Chemistry and Chemical Technology [online] [20.03.2011]. Available at: https://chem21.info/info/1326987/

[7] Chemistry Handbook 21. Chemistry and Chemical Technology [online] [20.03.2011]. Available at: https://www.chem21.info/info/787330/

[8] Ivanov E.G. Seed germination with use of acoustic-cavitation technologies. Proceedings of XVth International scientific conference "Engineering for rural development", May 25-27, 2016, Jelgava, Latvia, 2016, pp. 278-284.

[9] Karn A., Arndt R.E.A., Hong J. An experimental investigation into supercavity closure mechanisms. Journal of Fluid Mechanics, vol. 789, 2016, pp. 259-284.

[10] Sivakumar M., Tang S.Y., Tan Kh.W. Cavitation technology - A greener processing technique for the generation of pharmaceutical nanoemulsions. Ultrasonics Sonochemistry, vol. 21, No 6, 2014, pp. 2069-2083.

[11] Matsuno Y., Fukushima Y., Matsuo Sh. etc. Investigation on temperature separation and flow behavior in vortex chamber. Journal of Thermal Science, vol. 24, No 2, 2015, pp. 149-154.

[12] Matsuo Sh., Matsuno Y., Fukushima Y. etc. Experimental Study on Temperature Separation in Vortex Chamber. Procedia Engineering, vol. 105, 2015, pp. 464-471. 